\title{
VAST PLANES OF SATELLITES IN A HIGH-RESOLUTION SIMULATION OF THE LOCAL GROUP: COMPARISON TO ANDROMEDA
}

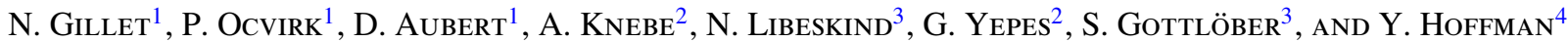 \\ ${ }^{1}$ Observatoire astronomique de Strasbourg, Université de Strasbourg, CNRS, UMR 7550, 11 rue de lUniversité, \\ F-67000 Strasbourg, France; nicolas.gillet@astro.u nistra.fr \\ ${ }^{2}$ Departamento de Física Teórica, Módulo, Universidad Autónomade Madrid, Cantoblanco E-28049, Spain \\ ${ }^{3}$ Leibniz-Institute für Astrophysik Potsdam (AIP), An der Sternwarte 16, D-14482 Potsdam, Germany \\ ${ }^{4}$ Racah Institute of Physics, Hebrew University, Jerusalem 91904, Israel \\ Received 2014 October 16; accepted 2014 December 9; published 2015 February 5
}

\begin{abstract}
We search for vast planes of satellites (VPoS) in a high-resolution simulation of the Local Group performed by the CLUES project, which improves significantly the resolution of previous similar studies. We use a simple method for detecting planar configurations of satellites, and validate it on the known plane of M31. We implement a range of prescriptions for modeling the satellite populations, roughly reproducing the variety of recipes used in the literature, and investigate the occurrence and properties of planar structures in these populations. The structure of the simulated satellite systems is strongly non-random and contains planes of satellites, predominantly co-rotating, with, in some cases, sizes comparable to the plane observed in M31 by Ibata et al. However, the latter is slightly richer in satellites, slightly thinner, and has stronger co-rotation, which makes it stand out as overall more exceptional than the simulated planes, when compared to a random population. Although the simulated planes we find are generally dominated by one real structure forming its backbone, they are also partly fortuitous and are thus not kinematically coherent structures as a whole. Provided that the simulated and observed planes of satellites are indeed of the same nature, our results suggest that the VPoS of M31 is not a coherent disk and that one-third to one-half of its satellites must have large proper motions perpendicular to the plane.
\end{abstract}

Key words: galaxies: dwarf - galaxies: kinematics and dynamics - Local Group

\section{INTRODUCTION}

The discovery of the planar distributions of satellite galaxies around the Milky Way (MW; Lynden-Bell 1976; Kunkel \& Demers 1976) and Andromeda (Koch \& Grebel 2006; McConnachie \& Irwin 2006; Ibata et al. 2013; Conn et al. 2013) is regarded as a new challenge to galaxy formation theory in the context of the standard model of cosmology $\Lambda \mathrm{CDM}$ (Kroupa et al. 2005). Using Pan-Andromeda Archaeological Survey (PAndAS), Ibata et al. (2013, hereafter I13) and Conn et al. (2013) found that among the 27 known satellites of Andromeda, 15 are located within a very thin, extended plane (with a thickness of $12.6 \pm 0.6 \mathrm{kpc}$ and about $200 \mathrm{kpc}$ in radius). Moreover, they estimate, from the radial velocities, that 13 are co-rotating. Shaya \& Tully (2013) find that of the 12 remaining satellites, 8 sit on a second plane roughly parallel to that found by I13. While such planar distributions of satellites are not impossible to find in $\Lambda \mathrm{CDM}$ simulations (Aubert et al. 2004; Libeskind et al. 2005; Kang et al. 2005; Zentner et al. 2005; Libeskind et al. 2007; Deason et al. 2011; Libeskind et al. 2009), their frequency and quantitative resemblance with the observed I13 vast planes of satellites (VPoS) are hotly debated. Bahl \& Baumgardt (2013) investigated the incidence of planar alignments of satellite galaxies in the Millennium-II simulation and concluded that vast, thin planes of dwarf galaxies, similar to that observed in the Andromeda galaxy (M31), occur frequently in $\Lambda \mathrm{CDM}$ cosmology. Shortly afterward, Ibata et al. (2014b) and Pawlowski et al. (2014) re-examined this simulation, accounting for the observed plane's extent, thickness, and abundance, and came to the opposite conclusion, that only $0.04 \%$ of galaxies possess planes as extreme as M31's. These studies were performed, "with the caveat that the Millennium-II simulation may not have sufficient mass resolution to identify confidently simulacra of low-luminosity dwarf galaxies", as duly noted by Ibata et al. (2014b, page 1): the semi-analytic modeling of Guo et al. (2013) differentiates normal galaxies from "orphans," the latter being systems whose parent sub-halo is no longer resolved. It is possible that many of these orphans are tidally disrupted, and hence that they are not directly comparable to the observed dwarf galaxies. In the present paper, we avoid this caveat by using a high resolution of the local group performed by the Constrained Local UniversE Simulation (CLUES) project, offering an improvement of a factor 15 in mass resolution with respect to the Millenium-II simulation, which allows us to resolve the satellites in the mass range of interest more consistently. This improvement comes however at the cost of volume, as we are left with only two host galaxies to study in the present paper. In Section 2, we present the simulation, the satellite population models used, and the method for detecting planes of satellites. In Section 3, we present the results and the detected planes, followed by a short discussion and our conclusions.

\section{METHODOLOGY}

This section describes the simulation used, the satellite population models, and the method for detecting and quantifying satellite alignments.

\subsection{The CLUES Simulation}

The simulation we use in this study was performed by the CLUES project (Gottloeber et al. 2010; Yepes et al. 2014), using GADGET2 (Springel 2005). It was run using standard $\Lambda \mathrm{CDM}$ initial conditions assuming a WMAP3 cosmology, i.e., $\Omega_{m}=0.24, \Omega_{b}=0.042, \Omega_{\Lambda}=0.76$ (Spergel et al. 2007), and uses a zoom technique, where a small, high-resolution region is embedded in a larger, low-resolution box providing the 
large-scale cosmological context. The zoom region is about $2 \mathrm{~h}^{-1} \mathrm{Mpc}$ wide at $z=0$ and contains a Local Group analog, with a mass resolution of $\mathrm{m}_{\mathrm{dm}}=2.1 \times 10^{5} \mathrm{~h}^{-1} M_{\odot}$ for the highresolution dark matter particles and $\mathrm{m}_{\text {gas }}=4.42 \times 10^{4} \mathrm{~h}^{-1} M_{\odot}$ for the gas. The feedback and star formation prescriptions of Springel \& Hernquist (2003) were used. For more details we refer the reader to (Gottloeber et al. 2010). This simulation has been used to investigate a number of properties of galaxy formation at high resolution (Forero-Romero et al. 2011; Knebe et al. 2011a, 2011b; Libeskind et al. 2011a, 2011b) and reionization studies (Ocvirk et al. 2013, 2014). Besides being a wellstudied simulation, the advantage of this data set for the present study is twofold. First of all, it produces a fairly realistic Local Group at $z=0$ : the MW and M31 are in the correct range of separation and total virial mass: $5.71 \times 10^{11} M_{\odot}$ for the MW and $7.81 \times 10^{11} M_{\odot}$ for M31 at a virial radius of $220.4 \mathrm{kpc}$ and $244.58 \mathrm{kpc}$, respectively (Table 2 of Libeskind et al. 2010). Also a cluster of roughly the size of Virgo is found some $12 \mathrm{Mpc}$ away from the simulated LG. In the rest of the paper, and for the sake of clarity, we will refer to the simulated galaxies as LGa and LGb, respectively, while MW and the M31 will refer to the real galaxies. Second, its mass resolution in the zoomed region allows us to resolve $\mathrm{M}_{\text {halo }}=4.2 \times 10^{6} \mathrm{~h}^{-1} M_{\odot}$ halos, which is comfortably below the expected or measured mass range of the satellite population of M31, and 15 times smaller than the twenty-particle halos of the Millenium-II simulation. The dark matter catalogs are produced by the Amiga halo finder (Gill et al. 2004; Knollmann \& Knebe 2009), and no halos that are contaminated with low-resolution particles are found within the volume considered here and thus only halos fully resolved by the lowest mass particles are used in our analysis. These $z=0$ halo catalogs give the mass, positions, and velocities of the dark matter halos. They will be used to analyze the properties of the satellite populations obtained. The simulation was also post-processed with the radiative transfer code ATON (Aubert \& Teyssier 2008, 2010) in order to compute a reionization redshift for each halo, which will be used in our satellite population models. This is described in detail in Ocvirk et al. (2014), which also used the results of this post-processing to study the correlation between present-day satellite positions and their reionization histories.

\subsection{Spatial Selection}

In order to be able to make the comparison of the simulation with the observed plane of satellites as direct as possible, we first perform a spatial selection of our halo populations as close as possible to the PAndAS volume. We also explore a different, slightly wider volume, and finally consider a spherical volume.

1. PAndAS: our first volume is a PAndAS-like volume around the host galaxy. The line of sight is taken along the line linking $\mathrm{LGa}$ to $\mathrm{LGb}$, and we fixed the distance of the observer at $780 \mathrm{kpc}$. The galaxy LGa will be the observer of LGb and vice-versa. The PAndAS area is modeled by a circular area of $12^{\circ}$ around Andromeda and because of the contamination due to Andromeda's stellar disk, satellites in the central 2.5 are rejected. Also detected satellites are constrained to $500 \mathrm{kpc}$ from the host forward and backward. We do not consider the extension of the survey around M33, which contains two satellites in the observations.

2. PAndAS-bis: here we will consider a modified PAndAS volume. The distance of the observer is increased to $1200 \mathrm{kpc}$, the outer angle limited to $10^{\circ}$ and the inner to $2 \circ$. This volume, larger than the original PAndAS volume, allows us to probe other configurations of the satellite population.

3. Spherical: our third volume is a simple sphere around the host, and therefore there is no line of sight. Satellites have to be closer than $500 \mathrm{kpc}$ and further than $50 \mathrm{kpc}$ from the host. This type of volume is similar to that chosen by Bahl \& Baumgardt (2013) using the Millenium-II simulation, which is why we include it in this study. This simple volume also allows us to explore the spatial distribution of satellites of the simulation more systematically, without the possible bias induced by the line of sight.

We now turn to modeling the satellite population of our simulated galaxies.

\subsection{Satellite Population Models}

The PAndAS survey only detected the 27 brightest satellites, while our simulation counts thousands of dark matter halos around each galaxy. Therefore, we need to find out which of our dark matter halos will be the 27 brightest, i.e., which ones will have the largest stellar mass. There is no real consensus on what shapes the properties of satellite populations, and as a result their modeling is still very uncertain. Therefore, we chose to explore a number of simple recipes, in an attempt to emulate at least partially the variety of models found in the literature. The initial halo catalog we used gives us for a sphere of $2 \mathrm{Mpc}$ around LGa and LGb, 5563 satellites and 6823, respectively, with their positions, velocities, dark matter masses at $z=0$, maximum dark matter masses throughout their assembly history $\mathbf{M}_{\max }$, stellar masses $\mathbf{M}_{\text {star }}$, and reionization redshift $\mathrm{Z}_{\mathrm{r}}$. We use this data and simple selections on these quantities or combinations thereof in order to mimic the basic behavior of a number of popular models.

First of all, we consider as surviving halos at $z=0$ only those having retained more than $5 \%$ of their maximum mass $M_{\max }$. Halos with larger mass loss are assumed to have experienced strong tidal disruption during their accretion on the host LGa or LGb and have lost their stars to the stellar halo of the host. This is similar to what is found in the literature (Busha et al. 2010; Ocvirk \& Aubert 2011; Macciò et al. 2010). Tests indicate that this criterion does not have a strong impact on our results. We also keep as satellites only those sub-halos which are gravitationally bound to the host. Then we select the $N_{\text {sat }}$ brightest halos (we will consider samples of $N_{\text {sat }}=25,27$, $30,35,50,100$, and 150 satellites) according to simple stellar content modeling using physically motivated criterions. The five models we considered are listed below. We do not focus on the absolute stellar mass content given by these models, but only use them in a relative manner, so as to determine the brightest $N_{\text {sat }}$.

1. $\mathrm{M}_{\text {star }}$ : the CLUES hydrodynamical simulation we used spawns star particles using the Springel \& Hernquist (2003) formalism. Therefore a stellar mass $\mathbf{M}_{\text {star }}$ can be computed for all dark matter halos. However, the properties of low-mass satellites populations are notoriously difficult to reproduce even with high-resolution hydrodynamical runs. Moreover, the simulation used a uniform UV background at reionization and therefore does not account for local inside-out effects such as shown in Ocvirk et al. (2013, 2014). Therefore we decided to explore several modeling alternatives.

2. $M_{z=0}$ (simple abundance matching): here we assume that the brightest satellites should be the most massive ones at $z=0$. This is the basic underlying assumption of 
the abundance matching technique, widely used in semianalytical modeling. This assumption is supported by the results of, e.g., Brook et al. (2014), where the stellar mass is taken to be a monotonic function of halo mass at $z=0$, but challenged by other groups (Sawala et al. 2014) because of the stochasticity of star formation at the mass scale of the faint M31 and MW satellites.

3. $Z_{r}$ (reionization reshifts): For each satellite we computed the redshift of last reionization using the results of the radiative transfer post-processing of Ocvirk et al. (2014). Reionization is thought to be one of the main causes of the low efficiency of star formation in low-mass satellites, as suggested for instance by Brown et al. (2014). Very often semi-analytical models account for reionization by shutting down star formation in low-mass halos (Koposov et al. 2008; Busha et al. 2010; Ocvirk \& Aubert 2011) at $\mathrm{z}_{\mathrm{r}}$. Therefore one could expect that the halos with the latest reionization redshift $z_{r}$ will be the brightest.

4. $\mathbf{M}_{\max }$ : according to Gnedin (2000), the effect of reionization on the baryonic fraction inside dark matter halos is a function of mass, and the transition between sterile and UV-immune halos takes place over two decades in mass. Therefore one could expect that the total stellar mass of a satellite progenitor halo is mostly tied to the maximum mass $\mathrm{M}_{\max }$ it has been able to reach throughout its life. Under this assumption the brightest satellites would be the one with the larger $\mathrm{M}_{\max }$, even if they are not the most massive at $z=0$. This is similar to the assumptions of Moster et al. (2013) for satellite galaxies.

5. $\mathrm{Z}_{\mathrm{r}} \mathrm{M}_{\text {max }}$ : the last model is an attempt at accounting for the mass scale at which halos become UV immune, and the variety of reionization histories of lower mass halos. For instance, (Pawlik et al. 2013) showed that halos more massive than $10^{9} M_{\odot}$ are insensitive to ionizing radiation. Therefore all halos that have grown beyond this threshold must have stars. On the other hand, the remaining less massive halos have seen their star formation history truncated at reionization. Under these assumptions, we select the brightest satellites as the halos with $\mathrm{M}_{\max }>10^{9} M_{\odot}$ (these are 10 for LGa and 9 for LGb), completed with the satellites with the latest $\mathrm{z}_{\mathrm{r}}$ to obtain a sample of $N_{\text {sat }}$ satellites.

We do not focus on the absolute stellar mass content given by these models, but only use them in a relative manner, so as to determine the $N_{\text {sat }}$ brightests. This modeling does not yield independent populations. Indeed, they will have some fraction of their satellites in common, depending on the number of bright satellites $N_{\text {sat }}$ considered. Here we do not try to tune our models to reproduce the properties of the observed population other than their number. This is notoriously complex, and beyond the scope of this paper. Instead, their rather different outcomes demonstrate the range of properties allowed for the model population. For instance, Figure 1 shows the radial distributions obtained within a PAndAS volume for our five models and the whole population of dark matter halos for LGa and LGb. First of all, we note that the two galaxies exhibit different satellite populations. Indeed the LGa satellite system is, in most of the cases, more extended than the LGb system. Besides, all models relying on $\mathrm{M}_{\mathrm{star}}, \mathrm{M}_{\mathrm{z}=0}$ or $\mathrm{M}_{\max }$ are too concentrated, while the $Z_{r}$ model is too extended, because of the typical inside-out reionization process described in Ocvirk \& Aubert (2011); Ocvirk et al. (2013) and Ocvirk et al. (2014): a late $\mathrm{z}_{\mathrm{r}}$ selection yields more remote halos. Finally, the $\mathrm{z}_{\mathrm{r}} \mathrm{M}_{\max }$ model, while doing slightly better, is still is not a great fit to the observations. Even without a rigorous Kolmogorov-Smirnov test, it is clear that none of our models reproduces the observed distribution very well. This misfit is not necessarily caused by our modeling: fairly large variations in the radial distribution of a galaxy of a given mass are expected due to cosmic variance, as a result of different mass assembly histories. For instance, Lunnan et al. (2012) shows, using the Aquarius simulations, that the radius containing half of the satellites can vary from $50 \mathrm{kpc}$ to $120 \mathrm{kpc}$ within the six MW realizations of the data set. We recall however that constrained simulations such as the one we used here exhibit smaller variance than the baseline cosmic variance Forero-Romero et al. (2011). How this affects the radial distribution of satellites is unclear. The radial distribution of the MW and M31 satellites are also dissimilar: although they are similar within $100 \mathrm{kpc}$, they differ dramatically in the outskirts of the halo (Yniguez et al. 2014), findings that support the idea that different mass accretion histories are reflected in the halo's dark matter profile (Deason et al. 2013). Moreover, the radial distribution is also affected by the physics considered: Libeskind et al. (2010) showed that at given mass resolution, hydrodynamics simulations produced satellites populations more concentrated than pure dark matter runs. The radial distribution is an important aspect nonetheless, because its concentration affects the probability of finding planar configurations of a given thickness. We will come back to this in Section 2.4.2 and show that we can actually correct the simulation for these differences when comparing with the observations. Given the strong impact of satellite population modeling on their spatial distribution, we can already expect that this modeling will also affect the properties of the planar configurations found.

\subsection{Finding Planes and Computing Their Significances}

\subsubsection{Satellite Plane Detection Method}

In order to find three-dimensional structures around the host galaxies we developed a simple method. This method can be applied regardless of the volume or the number of satellites of the model. We compute directly the number of satellites in a plane of a given thickness. We generate a random sample of planes. All the planes include the host galaxy and are defined by their normal vector. In order to fill uniformly and homogeneously the volume, 100,000 random planes are generated. We fix a thickness $2 \Delta$ for each plane. Then the distance to the planes of all the satellites of the model are computed. A satellite is define as inside the plane if its distance to the plane is smaller than $\Delta$. We fix the thickness in order to be able to detect the plane of Andromeda, $2 \Delta=40 \mathrm{kpc}$, i.e., slightly more than three time the rms of satellite distances to the plane as measured by I13. For each plane we obtain the number of satellites included inside a fixed thickness. This simple method can be applied, quickly, to every sample of satellites, observed or simulated, in the same manner.

\subsubsection{Computing Significance: Positions}

The plane detection algorithm returns the plane with the largest number of satellites (which we will refer to as the "maximum plane"), along with the number of satellites it contains $N_{\text {max }}$. It is tempting to compare directly the simulation's $N_{\text {max }}$ with that of the observed plane. In doing this we must however use extreme caution because of one important caveat: concentrated satellite populations tend to naturally have more satellites in any centered plane than extended satellite populations, 

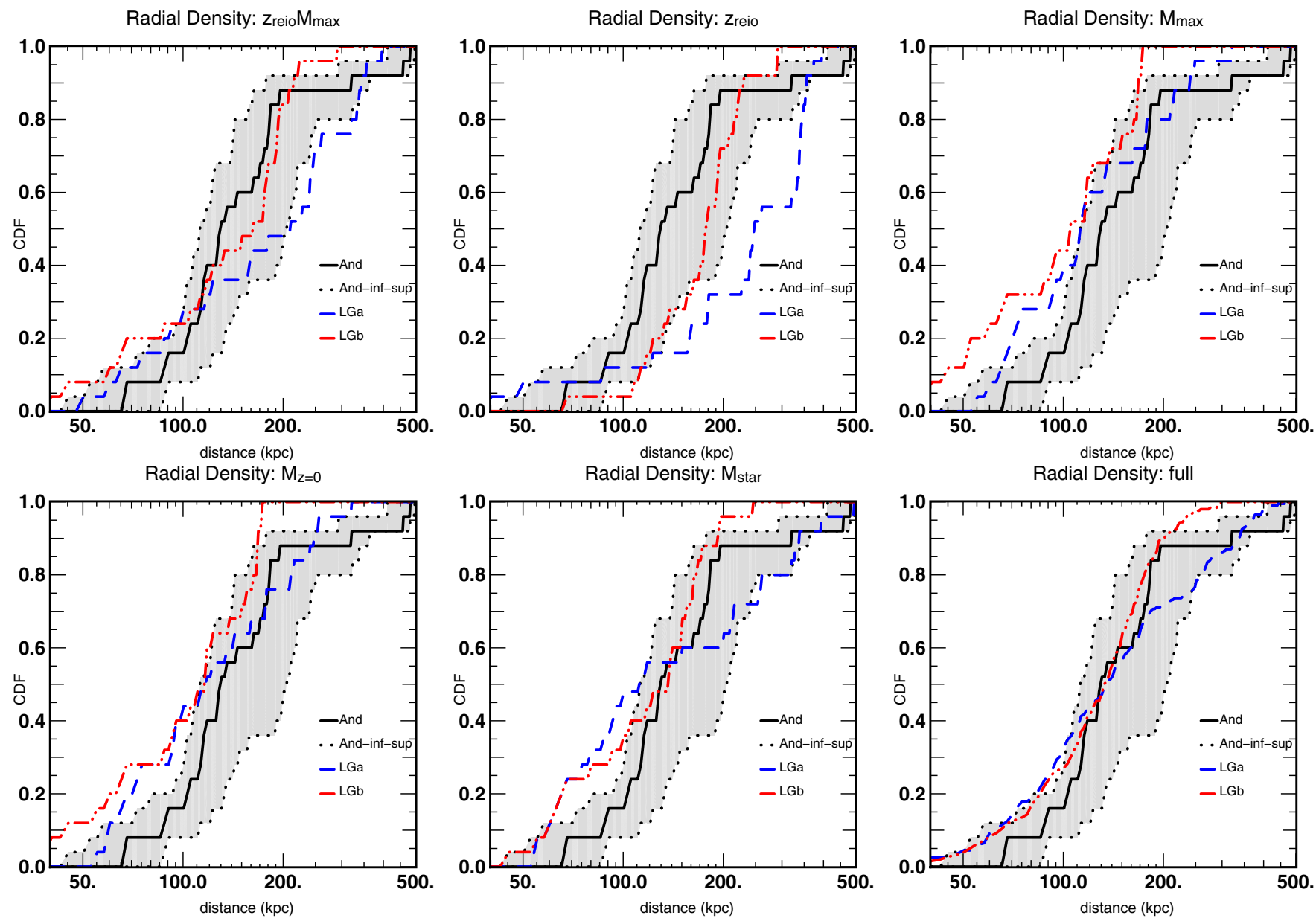

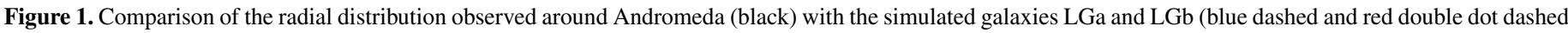

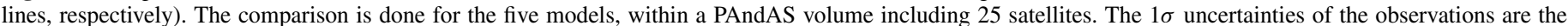

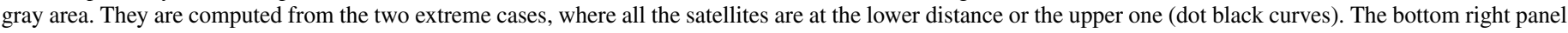
represent the radial distribution for the full sample of satellite halos, without any selection. It contains 245 satellites for LGa and 326 for LGb.

simply because they are more densely packed. Therefore, we also wish to quantify the "rareness" of the planar configurations we find. This quantity should allow us to tell whether a plane of $N$ satellites in the simulation is exceptional or not, given the radial distribution of the population. A good metric of this is the probability of obtaining a similar configuration in a fully random distribution of satellites. This is also the metric adopted by I13. We will refer to it as the "significance" of a plane. It is computed for a given detected plane, i.e., for a fixed volume (one of the three volumes defined in Section 2.2), and a fixed satellite population model, therefore a fixed radial distribution. To compute it we proceed as follows:

We randomly generate $N_{\text {sat }}$ satellites, respecting the radial distribution of the model, included in the volume of selection, spherical or PAndAS. We apply our maximum plane detection method to this new sample. This is done 100,000 times, with each realization producing a different $N_{\max }$. Therefore we obtain a probability distribution function (hereinafter pdf) of the number of satellites $N_{\max }$ in the maximum plane for the fixed radial distribution. The Figure 2 shows the $N_{\max }$ probability distributions for our five models applied to both LGa and LGb galaxies, for 25 satellites in a PAndAS volume. In both panels the $N_{\max }$ probability distribution of Andromeda (black) is also shown. The shift between the curves is induced by the radial distributions; when the satellite radial distribution is more concentrated, it is easier to find planes with a high number of satellites. We note that the pdf's are very peaked, with an average number of satellites in the maximum plane of about 10 satellites. Using these pdf's, we can compute the $p$ value of a plane of $k$ satellites as the probability of a random satellite system to host a plane with $k$ satellites or more. We will refer to this probability as the positional or spatial $p$ value:

$$
p_{\mathrm{pos}}=p(X \geqslant k)=\sum_{k}^{N_{\mathrm{sat}}} \mathrm{pdf} .
$$

\subsubsection{Computing Significance: Velocities}

An aspect making the VPoS of I13 even more striking is the apparent co-rotation of the plane. We can include this property in our definition of the significance. Let us consider a plane containing $N_{p}$ satellites. Then we assume that the direction of rotation of a satellite in the plane is equiprobable. Therefore the probability for one satellite to rotate one way or the other is the same as making head or tail when flipping a coin, which follows a binomial distribution. Therefore the probability of finding $k$ satellites rotating in the same direction is given by a binomial distribution. We will refer to this probability as the kinematic or 

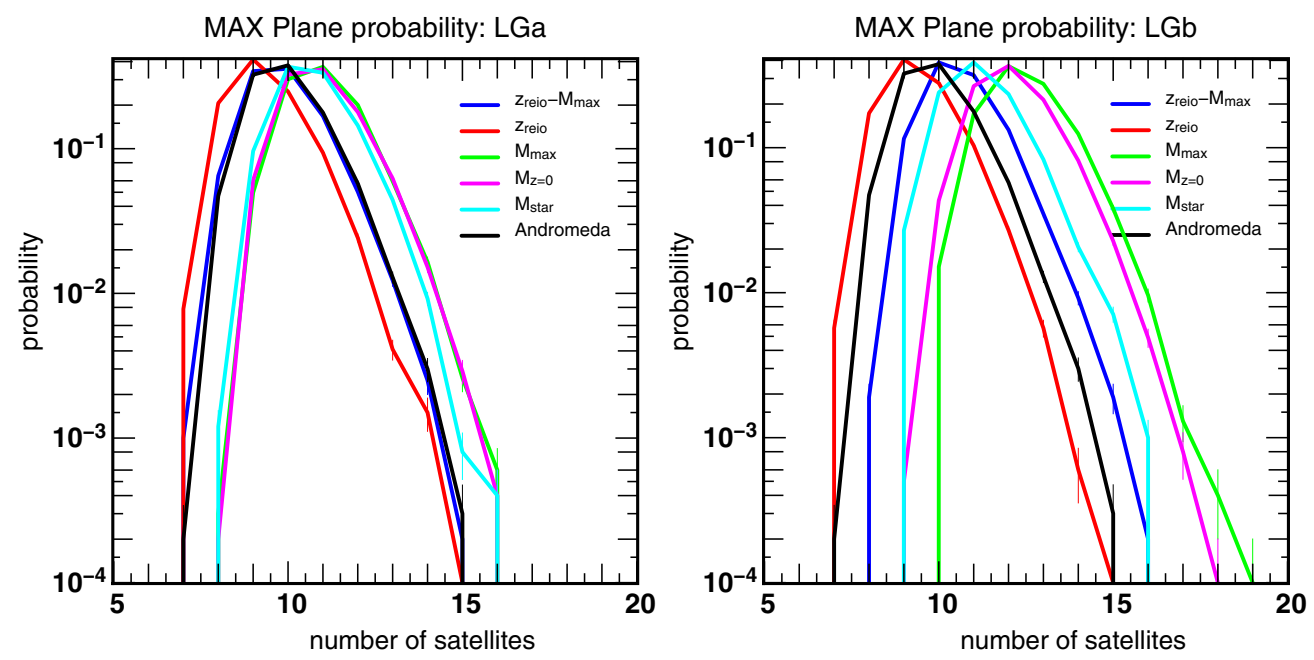

Figure 2. Probability distribution of number of satellites in the maximum plane, for the 10 samples of 25 satellites in a PAndAS volume. On the left panel the five samples around LGa, and on the right, the five around LGb. The color code for the different cases tested. The black curve on each panel is the probability distribution found for the observed radial distribution of Andromeda.

co-rotation $p$ value:

$$
p_{\text {kin }}=p(X \geqslant k)=2 \times \sum_{i=k}^{N_{p}} p(i)
$$

with

$$
p(i)=\left(\begin{array}{c}
N_{p} \\
i
\end{array}\right) \lambda^{i}(1-\lambda)^{N_{p}-i} .
$$

The probability $p_{\text {kin }}$ of finding $k$ or more co-rotating satellites in a plane of $N_{p}$ satellites is defined by Equations (2) and (3), taking $\lambda=0.5$. We multiply by 2 because we do not fix a preferential rotation; both are possible. With these conventions, $p_{\text {kin }}(k)$ only has a meaning for $k \geqslant N_{p} / 2$.

\subsubsection{Validation on the Observed VPoS of M31}

Here we validate our plane detection method by trying to detect the known plane of Andromeda (I13). We compiled the M31 satellite data by taking the $(1, b)$ coordinates from McConnachie \& Irwin (2006), the distances from Table 2 of Conn et al. (2012), and the velocities from Table 5 of Collins et al. (2013). Figure 3 shows the maximum plane detected by our method. We find 14 satellites in a plane of $40 \mathrm{kpc}$ thickness, with 12 co-rotating satellites. Here we do not take into account the distance uncertainties. Because of this, we do not find exactly the same plane as detected by I13. Indeed I13's plane hosts one additional satellite, AND III. This detail set aside, our method reliably recovers the existing plane of satellites of Andromeda.

The two last lines of Table 1 present our computation of the $p$ values for this detection of Andromeda's plane, along with the $p$ value published by I13. This configuration of 14 satellites in a plane has the probability of occurring of $1.6 \%$ assuming a random distribution. Accounting for the 12 co-rotating satellites, the total probability is $0.0208 \%$. I13 compute by Monte Carlo the probability for a plane of 15 satellites with 13 co-rotating in a sample of 27 satellites. They find $p$ values of $0.13 \%$ for the planar structure and $0.74 \%$ for the co-rotation, which make a total probability of $0.00096 \%$. The difference we find with respect to I13 is mainly due to AND III, which is included in I13's plane, but not in ours, due to a slightly different formulation of plane membership. If we use the probability density function we computed for the observed radial distribution and for 15 satellites, we find a probability of $0.33 \%$ for the planar structure. Multiplied by the probability of co-rotation of 13 satellites $(0.75 \%)$, we find a probability for this structure of $0.0024 \%$ to occur in a random population, which is more compatible with the estimation of I13.

Having described the simulation and plane detection method and validated the latter, we move on to searching planar configurations of satellites in the simulated galaxies.

\section{RESULTS}

In this section we apply our plane detection method to our model satellite populations in the three volumes considered.

\subsection{Planes of Satellites in the Simulation: 25 Satellites, PAndAS Volume}

We apply the method for all the satellite population models around the simulated host galaxies LGa and LGb. The maximum planes found for $\mathrm{LGa}$ and $\mathrm{LGb}$ are presented in Table 1. Column 3 of Table 1 gives $N_{\max }$, the number of satellites found in the maximum plane, of thickness $2 \times \Delta=40 \mathrm{kpc}$, with a selection of 25 satellites in a PAndAS area.

First, we find strong differences between LGa and LGb. Indeed, the maximum plane around LGb contains more satellites than LGa's. We know already that the two host galaxies have intrinsically different satellite populations. It can readily be seen in Figure 1 that the radial density profiles of $\mathrm{LGa}$ and $\mathrm{LGb}$ are different. The distribution of satellites is more extended around LGa. This could be the cause for its lower number of satellites in the maximum plane.

The least populated plane contains 9 satellites, and 14 for the biggest one. It has to be noted that we do not fix the angle between the line of sight and the detected plane. Therefore we can detect planes in any orientation with respect to the line of sight, and indeed find planes with a variety of orientations, as shown by Column 6 of Table 1, which gives the angle between the line of sight and the normal vector of the planes. Due to the axial symmetry, a similar angle does not guarantee that two planes have the same 3D orientation.

We do not find planes containing more satellites than the observed plane of Andromeda, but there is one plane containing 

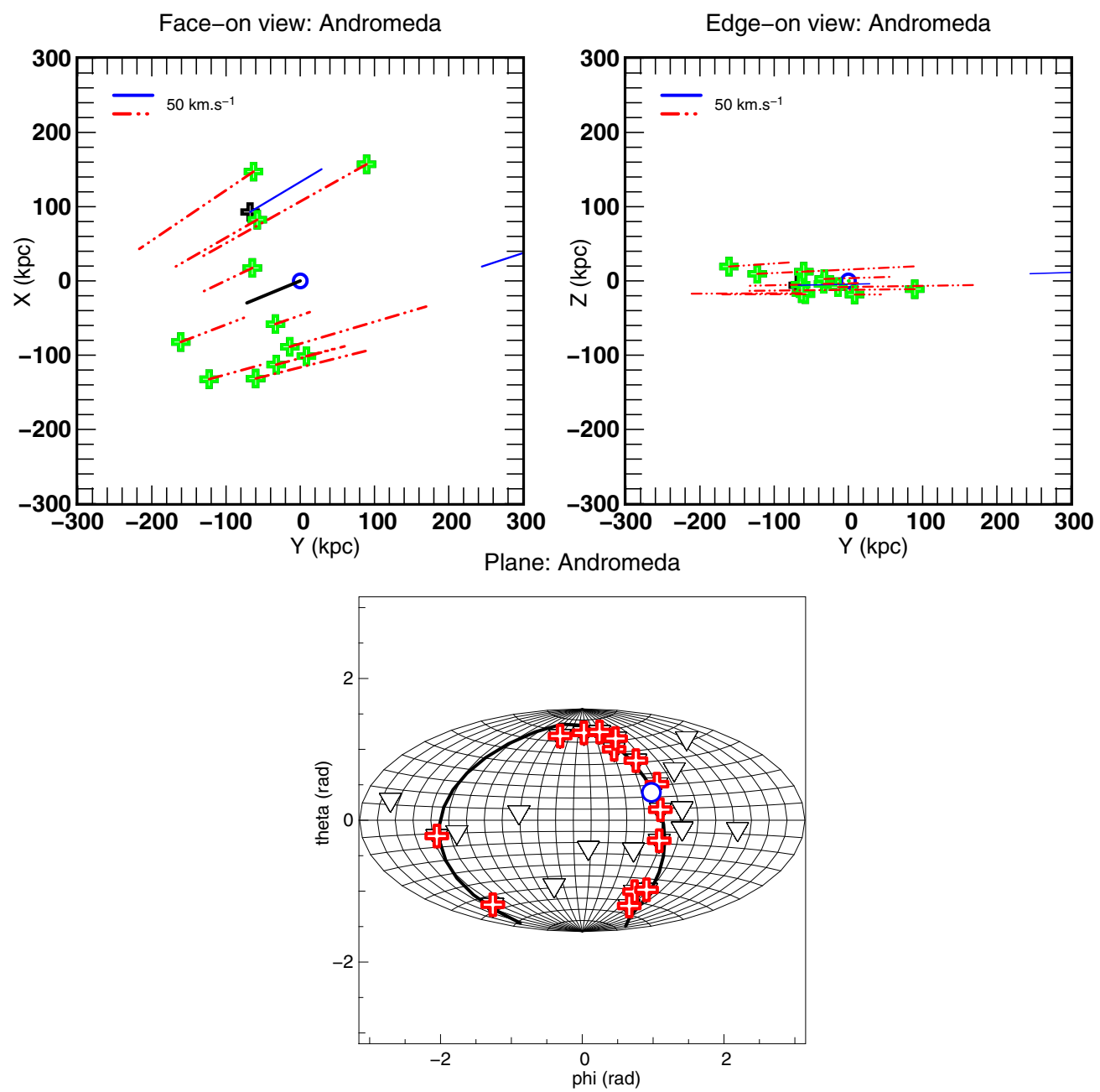

Figure 3. Observed plane of Andromeda (I13) as detected by our method. The face-on and edge-on view of the plane are presented in the top left and right panels. Only the satellites of the plane are shown (crosses), along with their velocities. The color of the satellites (green for the dominant rotation vs. black) and their velocities give their rotation directions. The blue circle with a black line shows the center of M31 and the direction toward the Milky Way. Bottom panel: the satellites of the plane are shown in red in an Aitoff-Hammer projection showing the positions of M31s satellites. The positions show where each object would appear in the sky if viewed from the center of M31. We detect 14 satellites, with 12 co-rotating. There are two additional satellites out of the boxes in the top left and right panels, one on the right and another on the left. We recall that only the line-of-sight velocity is known, and the plane is seen edge on. This alignment of the plane with the direction toward the Milky Way (blue circle) can also be seen in the right panel. The properties of this plane are given at the bottom of Table 1.

14 satellites. We compute the significance of the detections in order to do a proper comparison (see Section 2.4.2). In a first step we only consider the probability of finding a planar structure (Table 1, Column 7) assuming the radial distributions. Using only the spatial $p$ values there are no significant planes. ${ }^{5}$ The smallest spatial $p$ values are for the plane of 14 satellites of the $\mathrm{LGb} \mathrm{M}_{\max }$ model (16.77\%) and for the $\mathrm{LGb} \mathrm{z}_{\mathrm{r}}$ model $(13.91 \%)$ which contains only 11 satellites.

The effect of the radial distributions is again illustrated by the spatial $p$ values of the planes $\mathrm{LGb} \mathrm{z}_{\mathrm{r}} \mathrm{M}_{\max }$ and $\mathrm{z}_{\mathrm{r}}$ : both contain 11 satellites, but the former has a spatial $p$ value of $51.26 \%$ versus $13.91 \%$ for the latter. This means that for the radial distribution of $\mathrm{LGb} \mathrm{z}_{\mathrm{r}}$ it is more difficult to find a structure of 11 satellites than in $L G b z_{r} M_{\max }$. Indeed, the radial distribution of $L G b z_{r}$ is more dilute. We now proceed to include the kinematic properties of the planes in assessing their significance.

\footnotetext{
5 We recall that a $3 \sigma(5 \sigma)$ plane would have a $p$ value of $0.27 \%(0.00003 \%)$ for a Gaussian distribution of events. In the current paper we will arbitrarily refer to significant planes as having a $p$ value less than $1 \%$.
}

\subsubsection{Velocity in the Detected Planes}

An important aspect of the plane of Andromeda is the fact that 13 of the 15 satellites of the plane seem to co-rotate (I13). In the observations, only the line-of-sight velocity is accessible. In the simulation, all three components of the velocity are fully known. Therefore, it is possible to exactly determine the number of co-rotating satellites, unlike the observations. The Column 4 of Table 1 gives the number of co-rotating satellites for each detected plane and the $p$ value for the co-rotation $p_{\text {cor }}$ in Column 8 (see Section 2.4.3). The total significance or $p$ value of a given plane is the product of the two other $p$ values, spatial and co-rotation. It is given in Column 9 of Table 1. It is a more meaningful assessment of the significance of the planes found.

Here we consider that a total probability lower than $1 \%$ is a significant detection. Even if it is three decades above the observed planes, this still means that the detected planar configuration appears in only 1 in 100 realizations of a random satellite population. Therefore, finding such an alignment purely by chance is still very rare.

None of the simulation planes has a total significance as small as the observations. However, there is one case of significant 
Table 1

Detected Plane within the PAndAS Area with $N=25$ Satellites

\begin{tabular}{|c|c|c|c|c|c|c|c|c|c|c|c|}
\hline $\begin{array}{l}\text { (1) } \\
\text { Galaxy }\end{array}$ & $\begin{array}{c}(2) \\
\text { Model }\end{array}$ & $\begin{array}{c}(3) \\
N_{\max }\end{array}$ & $\begin{array}{c}(4) \\
N_{\text {cor }}\end{array}$ & 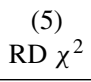 & $\begin{array}{c}(6) \\
\Phi\end{array}$ & $\begin{array}{c}(7) \\
p_{\operatorname{pos}}(\%)\end{array}$ & $\begin{array}{c}(8) \\
p_{\text {kin }}(\%)\end{array}$ & $\begin{array}{c}(9) \\
p_{\text {tot }}(\%)\end{array}$ & $\begin{array}{c}(10) \\
\sigma_{\|}(\mathrm{kpc})\end{array}$ & $\begin{array}{c}(11) \\
\sigma_{\perp}(\mathrm{kpc})\end{array}$ & $\begin{array}{c}(12) \\
\mathrm{L}_{\mathrm{LOS}} \min _{\min } \\
\end{array}$ \\
\hline \multirow{5}{*}{ LGa } & $\mathrm{z}_{\mathrm{r}} \mathrm{M}_{\max }$ & 9 & 5 & 1.15 & 54.3 & 93.75 & 100 & 93.7 & 187.4 & 13.4 & $0.63_{0.1}^{0.99}$ \\
\hline & $\mathrm{Z}_{\mathrm{r}}$ & 10 & 7 & 4.40 & 57.7 & 37.8 & 34.3 & 13.0 & 209.5 & 15.5 & $0.62_{0.37}^{0.96}$ \\
\hline & $\mathrm{M}_{\max }$ & 12 & 7 & 1.80 & 15.4 & 28.47 & 77.4 & 22.0 & 104.9 & 11.5 & $0.59_{0.32}^{0.86}$ \\
\hline & $\mathrm{M}_{\mathrm{z}=0}$ & 11 & 7 & 1.79 & 42.6 & 62.42 & 54.8 & 34.2 & 125.3 & 11.2 & $0.5_{0.28}^{0.76}$ \\
\hline & $\mathrm{M}_{\text {star }}$ & 12 & 7 & 1.29 & 62.5 & 20.31 & 77.4 & 15.7 & 160.7 & 13.9 & $0.6_{0.23}^{0.87}$ \\
\hline \multirow{5}{*}{$\mathrm{LGb}$} & $\mathrm{z}_{\mathrm{r}} \mathrm{M}_{\max }$ & 11 & 10 & 1.90 & 65.6 & 51.26 & 1.1 & 0.6 & 176.5 & 15.6 & $0.9_{0.56}^{1.55}$ \\
\hline & $\mathrm{z}_{\mathrm{r}}$ & 11 & 8 & 1.39 & 102.1 & 13.91 & 22.6 & 3.1 & 170.1 & 13.8 & $0.56_{0.22}^{0.95}$ \\
\hline & $\mathrm{M}_{\max }$ & 14 & 10 & 4.40 & 111.7 & 16.77 & 17.9 & 3.0 & 126.3 & 11.6 & $0.72_{0.42}^{1.03}$ \\
\hline & $\mathrm{M}_{\mathrm{z}=0}$ & 13 & 8 & 3.96 & 114 & 32.6 & 58.1 & 18.9 & 123 & 11.7 & $0.79_{0.42}^{1.25}$ \\
\hline & $\mathbf{M}_{\text {star }}$ & 11 & 7 & 2.82 & 130.6 & 72.61 & 54.8 & 39.9 & 116.8 & 15.4 & $0.8_{0.19}^{1.2}$ \\
\hline M31 & Observed & 14 & 12 & $X$ & 88.5 & 1.60 & 1.3 & 0.0208 & 154.7 & 12.5 & 1.47 \\
\hline M31 & I13 & 15 & 13 & $X$ & 89 & 0.13 & 0.74 & 0.00096 & 191.9 & 12.6 & 1.3 \\
\hline
\end{tabular}

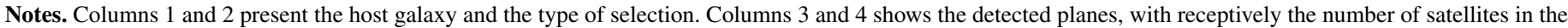

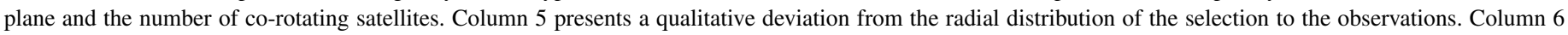

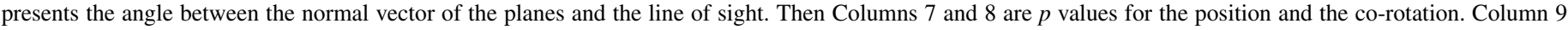

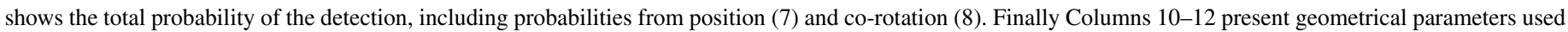

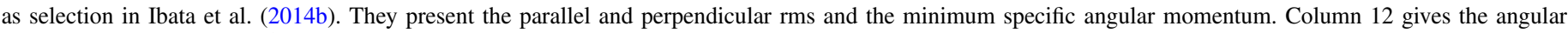

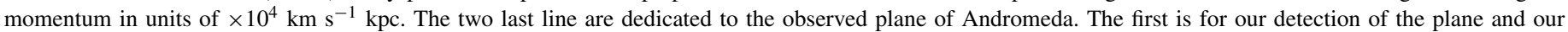
estimation of significance. The last second is the detection of I13 and their own estimation of $p$ values.

co-rotation. Indeed, in one of the planes, 10 satellites of a plane of 11 are rotating the same way, giving a probability of corotation of $1.1 \%$ (gray line in Table 1). However, the spatial $p$ value is $51.26 \%$ which gives a total probability of occurring at random for this plane of $0.60 \%$. This significant detection is around $\mathrm{LGb}$, for the $\mathrm{z}_{\mathrm{r}} \mathrm{M}_{\max }$ model.

This plane is an example of the fact that the probability of co-rotation permits planes that are not interesting in terms of planar structure to become significant. Another aspect is the sensitivity of the probability of co-rotation to small variations in the number of objects. Indeed, 7 satellites co-rotating over 11 give a probability of $54.8 \%$, while 8 over 11 give $22.6 \%$, and 10 over 11 is $1.1 \%$. A variation of one satellite can change the probability of co-rotation by more than $30 \%$. Figure 4 shows the face-on and edge-on view of the most significant plane. The face-on view illustrates the co-rotating nature of the plane.

We now explore the geometrical properties of the planes with the observations.

\subsubsection{Properties of the Most Significant Planes}

We use three additional parameters to compare the properties of the planes found in our simulation with the observations, in the spirit of Ibata et al. (2014b).

1. Plane thickness $\sigma_{\perp}$, computed as the perpendicular rms of the satellites' distance to the plane.

2. Plane size $\sigma_{\|}$, computed as the dispersion of galactocentric distances of the plane satellites.

3. $L_{\mathrm{LOS}}$ : the specific angular momentum for velocities evaluated along a line of sight. Therefore, the planes are not necessarily seen edge-on. To compute comparable values of $L_{\mathrm{LOS}}$, an edge-on line of sight has to be taken. Once the edge-on line of sight is fixed, we compute the median of the product between the velocities projected on the line of sight, and the distances in the plane to the host, as described in Ibata et al. (2014b). We perform this for 200 random lines of sight, and retain the average of $L_{\mathrm{LOS}}$ on the 200 lines of sight, with the maximum and minimum values, as given in Column 12 of Table 1.

We compute these parameters for our detection of Andromeda's plane, but we do not take into account AND XXVII in computing the parallel rms because the error is too large.

We compute these parameters for the maximum plane of all our models (Table 1 Columns 11-13). Our most significant plane contains 11 satellites with 10 that are co-rotating, giving a probability of occurring at random of $0.58 \%$ (Table 1 , gray line). For this plane, we find $\sigma_{\|}=176.5 \mathrm{kpc}, \sigma_{\perp}=15.6 \mathrm{kpc}$, and $\mathrm{L}_{\mathrm{LOS}}=1.55 \times 10^{4} \mathrm{~km} \mathrm{~s}^{-1} \mathrm{kpc}$ in the most favorable case. These values compare rather well with the observed plane.

\subsubsection{Conclusion for 25 Satellites in PAndAS Volume}

In this first exploration of the planes of satellites in our simulation, considering five different models for the satellite population, and using a pseudo-survey volume as close as possible to PAndAS, we find one rather exceptional plane, with a total probability of occurring in a random population lower than $1 \%$. This finding, in a simulation with only two major disk galaxies, suggests that the satellite population is not random and anisotropic, but highly structured. This plane is geometrically comparable to the observed plane in thickness and size. However, it contains only 11 satellites, 10 co-rotating, and therefore has a statistical significance (quantified by the total $p$ value) lower than the observed plane of I13. The simulation, while successfully reproducing some degree of structure in the satellite populations, does not yield satellite planes as extreme as the observed VPoS of M31.

In the rest of the paper, we will allow ourselves to modify the volumes and the number of satellites considered in order to analyze further the structure of the satellite populations of the simulation. 

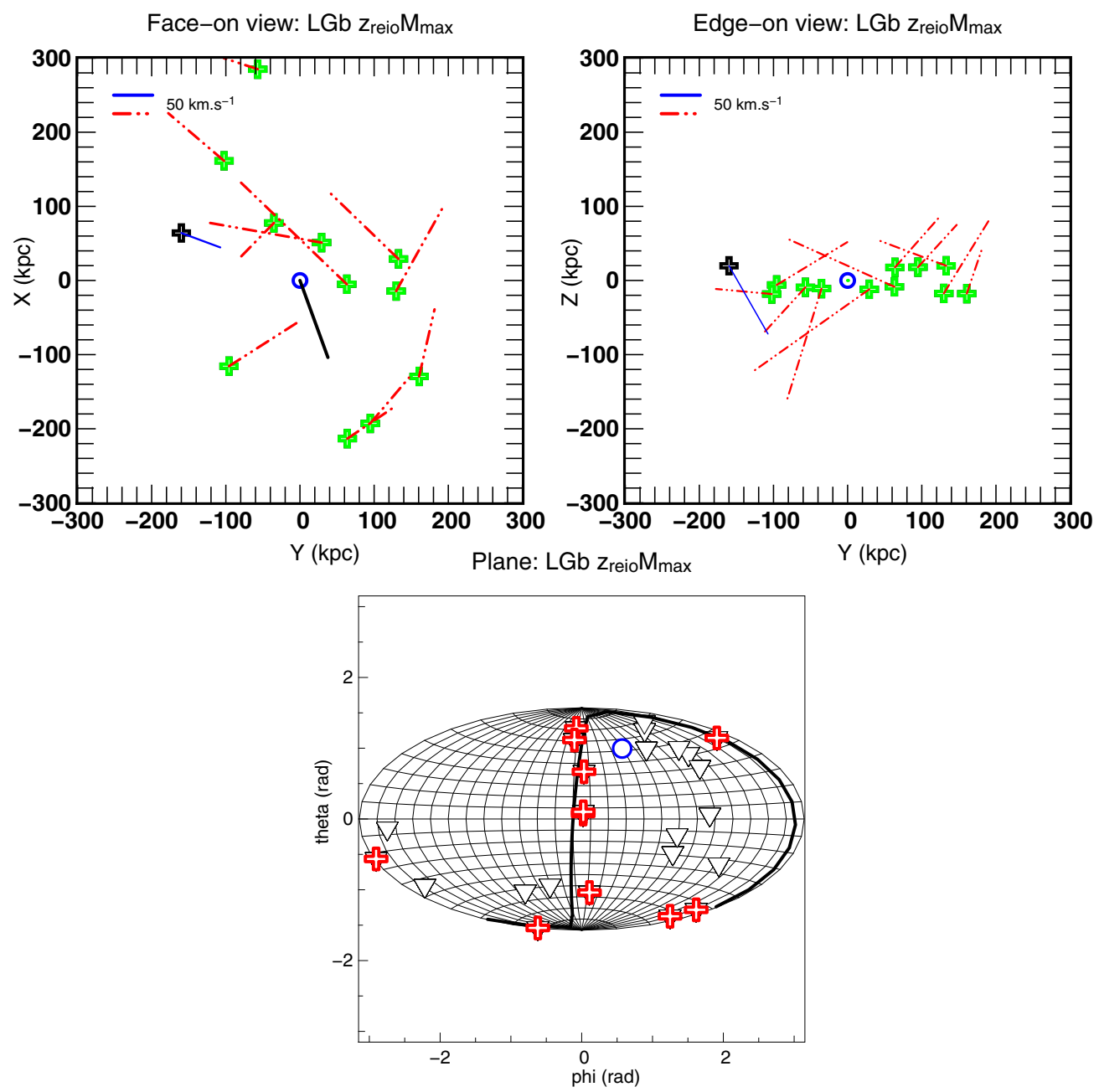

Figure 4. Planes around LGb, detected in the sample of 25 satellites of the $\mathrm{z}_{\mathrm{r}} \mathrm{M}_{\max }$ model in the PAndAS volume. The face-on and edge-on view of the plane are presented in the top left and right panels. Only the satellites of the plane are shown (crosses), along with their velocities. The color of the satellites (green for the dominant rotation vs. black) and their velocities give their rotation directions. The blue circle with a black line shows the center of LGb and the direction toward LGa. Bottom panel: the satellites of the plane are plotted in red in an Aitoff-Hammer projection as viewed from the center of LGb. The plane contains 11 satellites, with 10 co-rotating. Note that here the velocities are fully known, therefore we can see velocities pointing away from the plane, while this cannot happen with the observed plane due to the lack of proper motions. The orientation of the plane with respect to LGa (blue circle) can also be seen in the bottom panel. Note that the reference frame of the bottom panel is arbitrary, but remains fixed in all the figures of LGb's planes. This plane refers to row 6 of Table 1 ( $\mathrm{z}_{\mathrm{r}} \mathrm{M}_{\max }$ model).

\section{PLANES OF SATELLITES IN ALTERNATIVE VOLUMES}

The definition of the PAndAS volume imposes a line of sight and angular limits.

In this section we will explore the structure of the satellite population in a slightly extended PAndAS volume, which we will refer to as PAndAS-bis. The inner and outer limits of the cone are 2.5 ( $2^{\circ}$ in the PAndAS volume), and $12^{\circ}\left(10^{\circ}\right.$ in the PAndAS volume). We also change the MW-M31 distance, setting it to $1200 \mathrm{kpc}$, which is the distance between the two galaxies in the simulation. The resulting volume is larger than the PAndAS volume. Indeed, the projected inner and outer limits are now $42 \mathrm{kpc}$ and $207 \mathrm{kpc}$. Because the PAndAS-bis volume is larger, we will consider samples of 27 satellites, which is the number of satellites detected by I13 in the real PAndAS volume, which include an additional area around M33 compared to the quasi-circular area centered on M31. Therefore this volume allows us to investigate what could be found in slightly more remote satellite populations.

In a second step we will leave aside PAndAS and PAndASbis conical volumes and consider a simple spherical volume around the host. The sample consists of the satellites found in the 50-500 kpc shell. Finally, we will also explore more abundant satellite populations, by setting $N_{\text {sat }}=25,27,30$, $35,50,100$, and 150 satellites. Indeed, since the faintest MW satellites are still about 100 times fainter than the faintest M31 satellites known, one can only expect that future deeper surveys will discover new, fainter satellites hiding in the PAnDAS area. Therefore we take advantage of the current study and simulation to investigate the degree of structure this new population may display.

\subsection{PAndAS-bis Volume with 27 Satellites}

The Table 2 gives the properties of the planes detected in the PAndAS-bis volume with samples of 27 satellites.

First, as in Section 3.1, the two galaxies of the simulation give very different results. The detected planes are more abundant around $\mathrm{LGb}$, with 10 to 14 satellites in the planes, compared to $9-10$ in LGa. This reflects an intrinsical difference in the distributions of the satellites around the two galaxies: LGa's population is more extended spatially, making rich planes of a given thickness more rare. 
Table 2

Same as Table 1 for 27 Satellites in the PAndAS-bis Volume

\begin{tabular}{|c|c|c|c|c|c|c|c|c|c|c|c|}
\hline $\begin{array}{l}\text { (1) } \\
\text { Galaxy }\end{array}$ & $\begin{array}{c}(2) \\
\text { Model }\end{array}$ & $\begin{array}{c}(3) \\
N_{\max }\end{array}$ & $\begin{array}{c}(4) \\
N_{\text {cor }} \\
\end{array}$ & $\begin{array}{c}(5) \\
\mathrm{RD} \chi^{2}\end{array}$ & $\begin{array}{l}(6) \\
\Phi\end{array}$ & $\begin{array}{c}(7) \\
p_{\mathrm{pos}}(\%) \\
\end{array}$ & $\begin{array}{c}(8) \\
p_{\text {kin }}(\%)\end{array}$ & $\begin{array}{c}(9) \\
p_{\text {tot }}(\%)\end{array}$ & $\begin{array}{c}(10) \\
\sigma_{\|}(\mathrm{kpc})\end{array}$ & $\begin{array}{c}(11) \\
\sigma_{\perp}(\mathrm{kpc})\end{array}$ & $\begin{array}{c}(12) \\
\operatorname{L}_{\operatorname{Lax}} \\
\text { min } \\
\end{array}$ \\
\hline \multirow{5}{*}{ LGa } & $\mathrm{z}_{\mathrm{r}} \mathrm{M}_{\max }$ & 9 & 6 & 2.66 & 125.3 & 84.2 & 25.4 & 21.4 & 222.7 & 12.0 & $0.62_{0.09}^{1.0}$ \\
\hline & $\mathrm{Z}_{\mathrm{r}}$ & 9 & 6 & 7.88 & 126.3 & 47.3 & 25.4 & 12.0 & 266.1 & 13.3 & $0.77_{0.18}^{1.08}$ \\
\hline & $\mathrm{M}_{\max }$ & 10 & 7 & 0.54 & 129.0 & 90.2 & 17.2 & 15.5 & 125.0 & 14.6 & $0.60_{0.15}^{0.90}$ \\
\hline & $\mathrm{M}_{\mathrm{z}=0}$ & 10 & 6 & 1.10 & 136.7 & 82.0 & 37.7 & 30.9 & 178.3 & 11.7 & $0.79_{0.35}^{1.19}$ \\
\hline & $\mathbf{M}_{\text {star }}$ & 10 & 8 & 1.12 & 104.5 & 88.9 & 5.5 & 4.9 & 184.7 & 15.3 & $0.54_{0.26}^{0.72}$ \\
\hline \multirow{5}{*}{ LGb } & $\mathrm{z}_{\mathrm{r}} \mathrm{M}_{\max }$ & 14 & 11 & 1.11 & 55.0 & 0.55 & 2.9 & 0.016 & 176.3 & 14.1 & $1.23_{0.42}^{1.98}$ \\
\hline & $\mathrm{Z}_{\mathrm{r}}$ & 10 & 10 & 4.49 & 90.5 & 24.4 & 0.1 & 0.024 & 212.7 & 14.0 & $0.98_{0.55}^{1.49}$ \\
\hline & $\mathrm{M}_{\max }$ & 13 & 11 & 1.02 & 66.2 & 33.0 & 1.1 & 0.36 & 140.4 & 12.7 & $0.72_{0.40}^{0.99}$ \\
\hline & $\mathrm{M}_{\mathrm{z}=0}$ & 14 & 9 & 0.71 & 69.6 & 3.96 & 13.3 & 0.53 & 145.1 & 13.7 & $0.80_{0.40}^{1.38}$ \\
\hline & $\mathbf{M}_{\text {star }}$ & 12 & 7 & 0.76 & 49.6 & 45.8 & 38.7 & 17.7 & 123.8 & 15.4 & $0.76_{0.32}^{1.11}$ \\
\hline
\end{tabular}

We find four planes with probabilities to occur at random lower than $1 \%$, from which two are lower than $0.1 \%$ (gray and dark-gray lines in Table 2). We will now analyze the two most significant planes of $\mathrm{LGb}$, corresponding to models $\mathrm{z}_{\mathrm{r}} \mathrm{M}_{\mathrm{Max}}$ and $\mathrm{Z}_{\mathrm{r}}$. The LGb $\mathrm{z}_{\mathrm{r}} \mathrm{M}_{\text {Max }}$ plane contains 14 satellites with 11 corotating, while the $\mathrm{LGb} \mathrm{z}_{\mathrm{r}}$ plane only contains 10 satellites but all of them are co-rotating. These two planes are interesting because they are highly significant for two quite different reasons. The first has a high structural significance $\left(p_{\text {pos }}\right)$, while the second stands out due to its kinematic properties (100\% co-rotation).

Both planes seem to made up of groups of satellites. This is illustrated by the top left panel of Figure 5, featuring one large group of six satellites (around $y=100 \mathrm{kpc}, x=-100 \mathrm{kpc}$ ). The top right panel shows that the velocities of the group are mostly within the plane. There are however five satellites with strong out of the plane velocities, which will therefore get out of the plane on a short timescale. Therefore the nature of this plane is dual: it is mainly composed of one coherent group travelling together, plus a handful of additional satellites which are accidentally aligned with it at the time of observation or analysis. This apparent clumpiness of the satellites in the plane is not unlike the observed VPoS of M31: this can be seen by comparing the top left panel of Figure 3 and 5, at least for the $x<0$ half of the figure. The velocities on the other hand cannot be compared so easily since only the line-of-sight velocities are available in the observations. The structure of the other plane $\left(\mathrm{LGb} \mathrm{z}_{\mathrm{r}}\right)$ is shown in Figure 6: it consists of two groups of satellites, plus two accidentally aligned satellites. On the top left and right panel, one group has in-plane velocities, while the other group's velocities point perpendicularly to the plane. These two groups are internally coherent, but are otherwise unrelated. They form the backbone of the plane; the two additional satellites belonging to the plane are pure chance. The thick black line of the left panel of Figure 5 shows the direction to LGa. From this panel we can infer that the $\mathrm{LGb}_{\mathrm{r}}$ plane would display line-ofsight kinematics qualitatively similar to the observed $\mathrm{VPoS}$ if observed from LGa (i.e., receding on one side and approaching on the other side), yet it is clearly not a disk and the plane has no kinematical coherence as a whole.

In both cases, the planes are almost aligned with the other galaxy LGa, as shown by the bottom panel of Figures 5 and 6 . We also note that the satellites of the planes are not symmetrically distributed. Indeed most of the satellites are located in the near half rather than the far half with respect to the other galaxy, as in the observed VPoS. However we restrain from interpreting this since we have only two clear occurrences at hand.
As a preliminary conclusion, we see that the simulated galaxies feature planes of satellites which have some degree of similarity in richness and geometry with the observed VPoS of M31, although their $p$ values are not as low. These planes consist of one or more coherent satellite groups, although the groups themselves are unrelated. Therefore they are not coherent discs, even if they can appear as such if only line-of-sight velocities are available.

\subsection{Spherical Volume}

In this section we consider a spherical volume selection in order to explore the simulation in quest of a planar structure, without the possible bias due to the conical shape of the PAndAS and PAndAS-bis volumes. We will vary the sample size from 25 to 150 .

It is important to note that the samples are not independent. For example, the selection $\mathrm{z}_{\mathrm{r}}$ and $\mathrm{z}_{\mathrm{r}} \mathrm{M}_{\max }$ have a fair number of satellites in common in the 100 and 150 satellite samples.

In the 70 samples considered $(2 \times 7 \times 5)$ we find 12 planes with a probability of occurring at random lower than $1 \%$. The properties of the planes are presented in Table 3. Due to the finite size of the halo population, two different models can yield the same maximum plane. Indeed we find that among the 12 planes found, only 7 of them are unique. We present the results for the case of 100 satellites in a spherical volume in Table 4. In this case one significant maximum plane is detected twice (gray lines in Table 4), as indicated by the very similar properties of both lines and visual inspection. However, not all the satellites are the same in these two models, which is why small differences in the geometrical properties (Columns 10-12) are found. This plane has a strong structural significance, indeed, 32 satellites out of 100, one-third, are detected in a plane of $40 \mathrm{kpc}$ thick. The probability of occurring at random is $0.07 \%$ assuming the radial distribution of the $\mathrm{LGb} \mathrm{z}_{\mathrm{r}}$ model. Its kinematical coherence is rather weak, with 21 satellites out of 32 , two-thirds, that are rotating the same way, giving a probability $p_{\text {kin }}=11 \%$. This plane has, finally, a total probability of occurrong at random of $0.0077 \%$. Therefore, even if its $p$ value is not as low as the observed $\mathrm{VPoS}$ of M31, the structure and kinematics of the simulated satellite population is highly non-random. The plane is geometrically comparable to the observations with a $\sigma_{\|}$of $212 \mathrm{kpc}$ and a $\sigma_{\perp}$ of $13.3 \mathrm{kpc}$. However, it has a small specific angular momentum, with on average $\mathrm{L}_{\mathrm{LOS}}=0.75 \times 10^{4} \mathrm{~km} \mathrm{~s}^{-1} \mathrm{kpc}$ and a maximum of only $1.01 \times 10^{4} \mathrm{~km} \mathrm{~s}^{-1} \mathrm{kpc}$. 

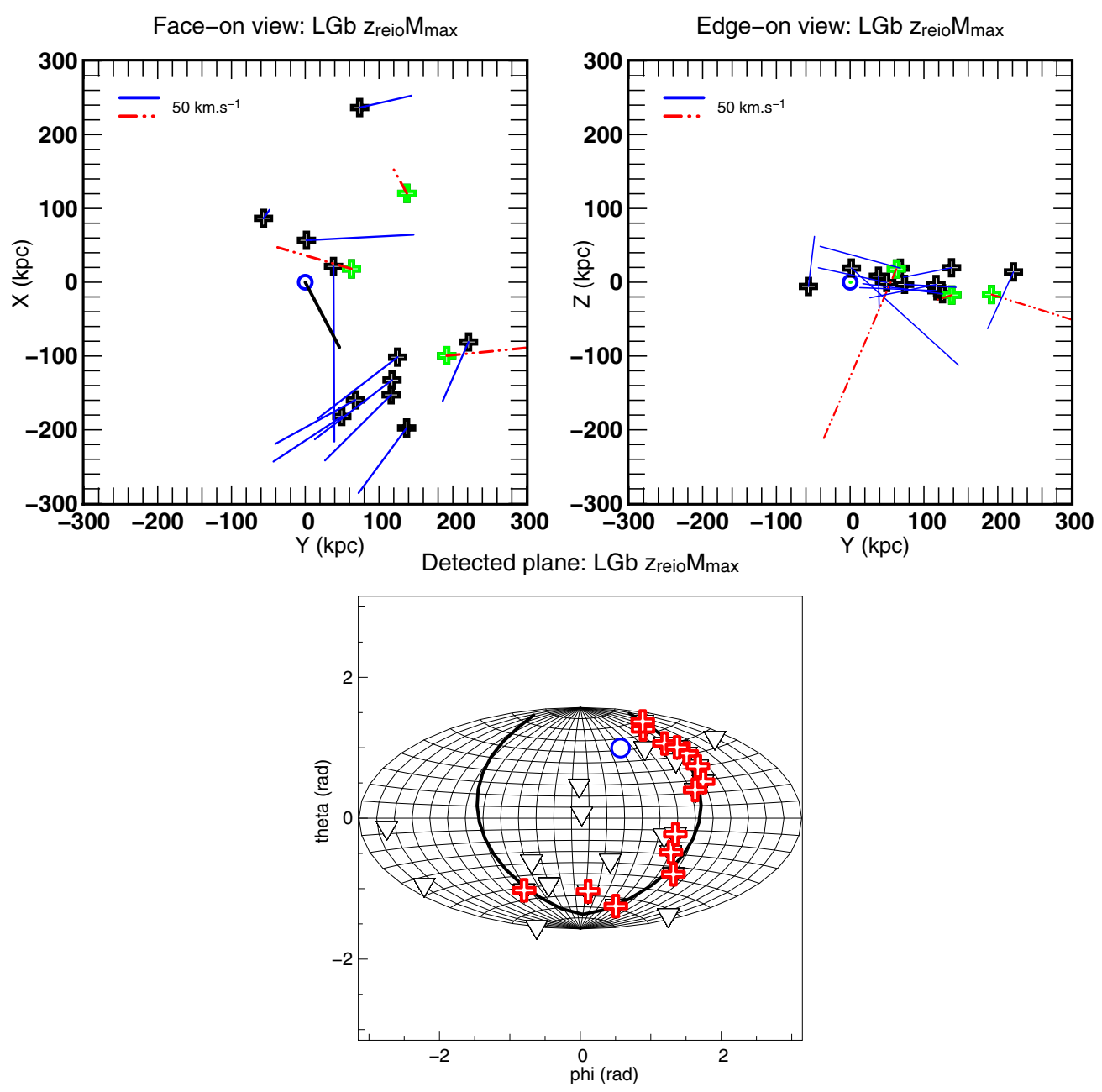

Figure 5. Plane of satellites around LGb, detected in the sample of 27 satellites of the $\mathrm{z}_{\mathrm{r}} \mathrm{M}_{\max }$ selection in the PAndAS-bis volume. The face-on and edge-on view of the plane are presented on the top left and right panels. Only the satellites of the plane are represented by crosses and segments indicating the in-plane component of the velocities. The color of the symbols code their direction of rotation, which can also be judged from the velocity vectors. Bottom panel: the satellites of the plane are plotted in red in an Aitoff-Hammer projection as viewed from the center of LGb. We detect 14 satellites, with 11 co-rotating. The bottom panel also shows the orientation of the plane with respect to LGa (blue circle). Note that the reference frame of the bottom panel is arbitrary, but remains fixed in all the figures of LGb's planes. This figure refers to the plane described in line 6 of Table 2.

Figures 7 show the face-on and edge-on view of this maximum plane, with the trajectories of the satellites in the top row, and the velocities at $z=0$ in the bottom row. Figure 7 relates to Table 4, line 2 (LGa model $z_{r}$ ). On the bottom left panel, visual inspection reveals a handful of a small coherent group of two to three satellites. On the bottom right, we estimate that a third of the 32 satellites of the planes have velocities pointing away from the plane, while the remaining two-thirds are well aligned.

The bottom left panel shows that the plane is very lumpy, with one large coherent group of satellites forming the bulk of the plane.

As in the previous section, this figure also shows that the plane would appear as roughly disk-like if only the line-of-sight velocities were considered (i.e., receding on one side of the host, and approaching on the other side).

\section{DISCUSSION}

\subsection{To Disk or not to Disk?}

The planes we find in the simulation do not reproduce exactly the observed properties of the VPoS but in a few cases they come quite close. The $\mathrm{z}_{\mathrm{r}} \mathrm{M}_{\max }$ model for the PAndAS-bis selection, for instance, is close to matching the observed VPoS, in richness, structure, and kinematics, as shown by Table 2 . We recall that in this simulation, the LGb galaxy which hosts the most realistic planes has a mass of only $7.81 \times 10^{11} M_{\odot}$, while the range for M31's mass goes up to $M_{300}=1.0-1.8 \times 10^{12} M_{\odot}$ (Watkins et al. 2010; van der Marel et al. 2012; Diaz et al. 2014), i.e., possibly two times larger than our simulated M31. It is very likely that this difference in mass affects our results: a more massive $\mathrm{LGb}$ would have a richer, more extended satellite system and larger angular momentum, potentially bringing the simulation in better agreement with the observations with respect to VPoS's. We plan to follow up on this by studying a new CLUES simulation in a forthcoming paper. Moreover, the fact that we find rich planes of satellites in the current simulation where only two relevant satellites systems can be studied (LGa's and LGb's) suggests that such structures are likely to be common. It is not clear why our small volume simulation features satellite planes not too dissimilar to M31's, whereas previous studies such as Ibata et al. (2014b) showed that they are rare. It could be due to numerical resolution, since our 

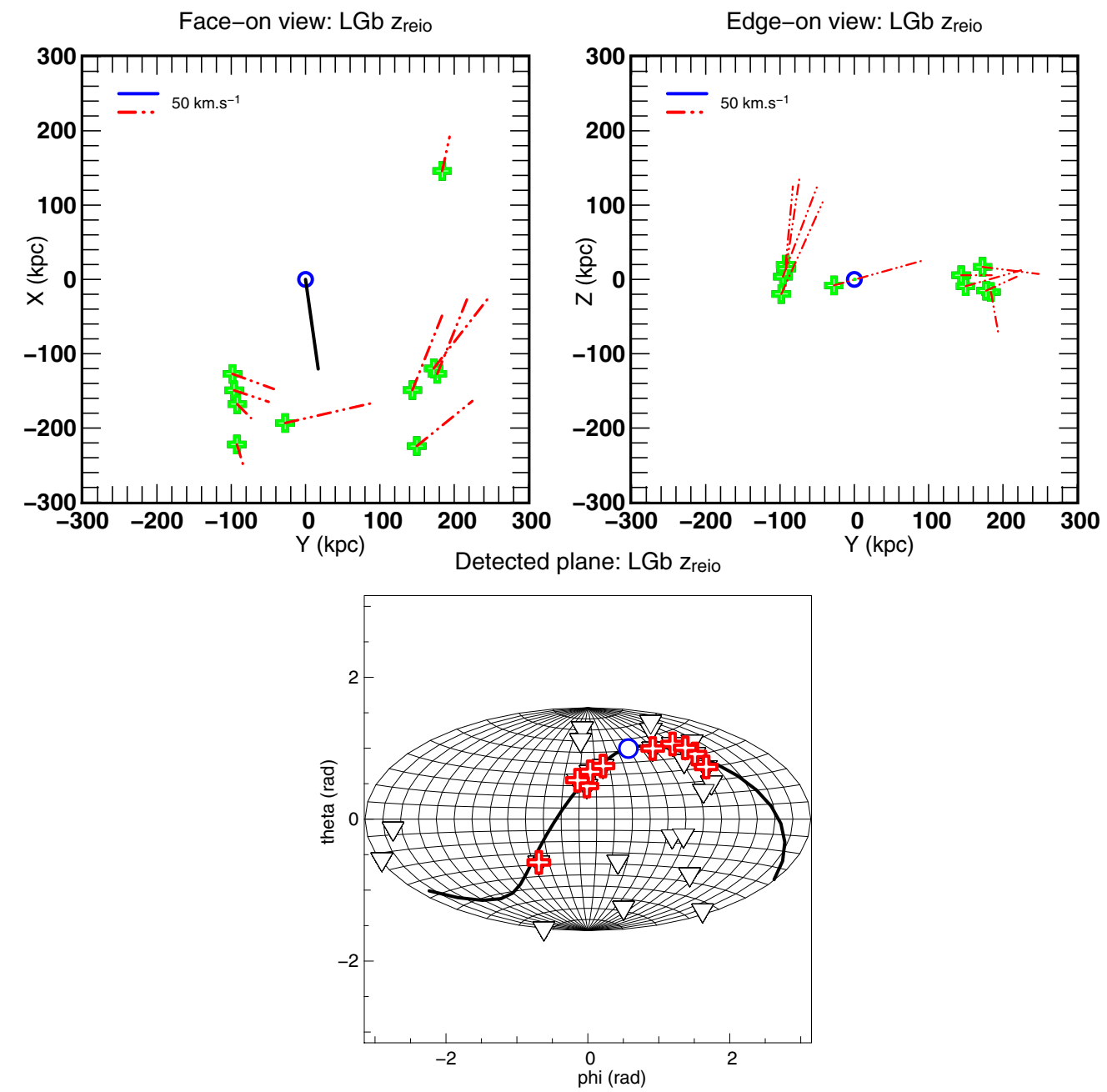

Figure 6. Planes around LGb detected in the sample of 27 satellites of the $\mathrm{z}_{\mathrm{r}}$ model in the PAndAS-bis volume. The face-on and edge-on view of the plane are presented in the top left and right panels. Only the satellites of the plane are shown (crosses), along with their velocities. The color of the satellites (green for the dominant rotation vs. black) and their velocities give their rotation directions. The blue circle with a black line shows the center of LGb and the direction toward LGa. Bottom panel: the satellites of the plane are plotted in red in an Aitoff-Hammer projection as viewed from the center of LGb. The plane contains 10 satellites, with 10 co-rotating. The bottom panel also shows the orientation of the plane with respect to LGa (blue circle). Note that the reference frame of the bottom panel is arbitrary, but remains fixed in all the figures of the LGb's planes. This plane refers to row 7 of Table $2\left(z_{r}\right.$ model).

simulation is 15 times better resolved in mass than Millenium-II. Do high-resolution simulations produce more significant planes of satellites than low-resolution runs? This remains to be shown. It could also be pure luck, but it is difficult to compare our results with those of Ibata et al. (2014b) since the number of satellites in the plane are different. It could also be an environmental effect, considering paired galaxies instead of isolated ones, as in Ibata et al. (2014b). This is not our favored explanation since Pawlowski \& McGaugh (2014) did not find this parameter to have a strong impact on satellite distributions. Our constrained simulation also captures by construction the environment of the LG on a larger scale, for instance with the proximity of a galaxy cluster. Forero-Romero et al. (2011) proposed that the environment may affect the mass assembly history of LG galaxies. Could it also affect the frequency of planar satellite configurations? To answer this will require comparative studies, such as the present work, on constrained versus unconstrained simulations of galaxy pairs. In several previous studies the VPoS of M31 is described as a rotating disk of satellites (Conn et al. 2013; Bowden et al. 2013; Pawlowski et al. 2014). One must recall, though, that only the line-of-sight velocities are currently available for these objects. Therefore the rotational support of the plane is unproven, and until proper motions are available, cannot be firmly assessed. Provided that the planes of satellites in our simulation and in M31 are of the same essence, our results provide interesting insight into this question. Indeed, the simulated planes are not coherent kinematical structures. They consist mostly a of a group of satellites forming the backbone of the plane (about half to two-thirds of the plane's satellites), which aligns by chance with a number of random satellites or in one case with another small group. While the main group is indeed a coherent structure resembling a group accretion event, and moves within the plane, the other chance-aligned satellites will fly out of the plane in a short timescale: about $150 \mathrm{Myr}$ assuming average perpendicular velocities of about $100 \mathrm{~km} \mathrm{~s}^{-1}$ and a plane thickness of $15 \mathrm{kpc}$. Therefore the current plane appears short-lived, but if its backbone (the main group) is long-lived, then we expect new satellites to randomly enter the plane while some others move out. In this respect, the plane may still be long-lived (supported by its main satellite group), but one-third to one-half of its satellites are non-permanent members. Therefore we consider the plane is half real (i.e., coherent) and half random, and this is the main result of this work. They nevertheless display velocity patterns characteristic 
Table 3

Significant Detected Planes within a Spherical Volume with $N_{\text {sat }}$ Going from 25 to 150

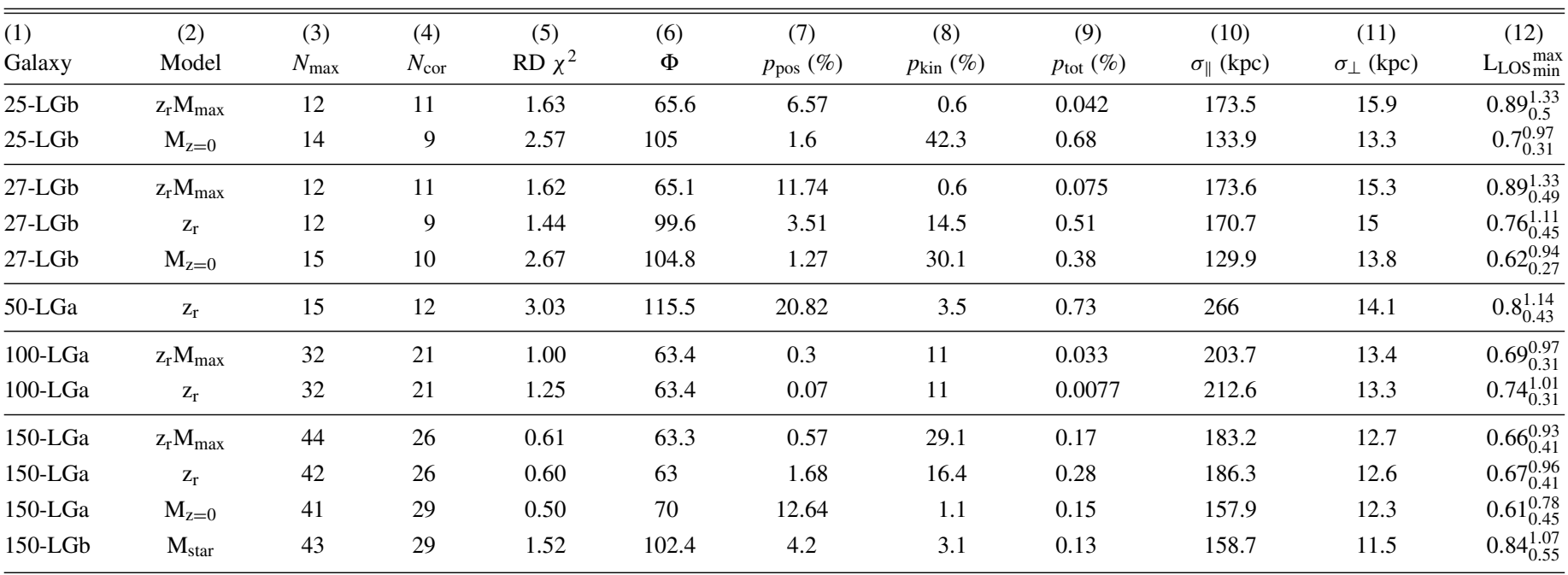

Notes. Column 1 presents the $N_{\text {sat }}$ with host galaxy and 2 presents the type of selection. Columns 3 and 4 shows the detected planes, with, respectively, the number of satellites in the plane and the number of co-rotating satellites. Column 5 presents a qualitative deviation from the radial distribution of the selection to the observations. Column 6 presents the angle between the normal vector of the planes and the line of sight. Columns 7 and 8 are the $p$ values for the position and the co-rotation. Column 9 shows the total probability of the detection, including probabilities from position (7) and co-rotation (8). Finally, columns 10, 11, and 12 present geometrical parameters used for selection in Ibata et al. (2014b). They present the parallel and perpendicular rms and the minimum specific angular momentum.
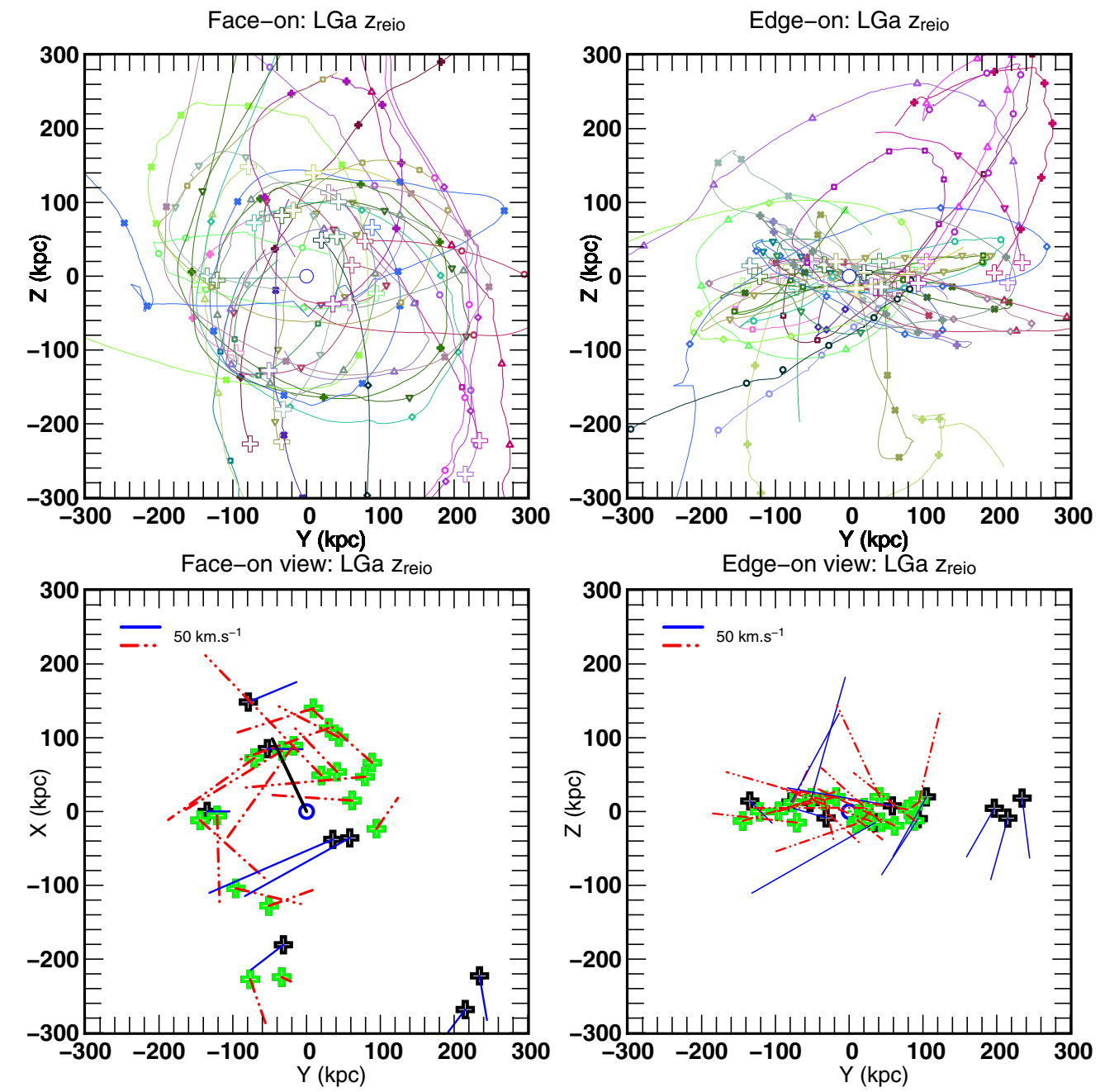

Figure 7. Significant planes for the LGa $\mathrm{z}_{\mathrm{r}}$ model for $N_{\text {sat }}=100$ satellites. The top row shows the trajectories seen face-on (left) and edge-on (right). The cross are the position at $z=0$, and the dots on the trajectories are separated by a $50 \mathrm{Myr}$ duration. The small hickups along some trajectories are due to problematic halo identifications during rapid evolution, such as galaxy mergers. They do not affect our results. The bottom row shows the plane's satellites' positions and velocities at $z=0$. The color of the satellites and their velocity vectors denote the direction of rotation. This plane refer to Table 4 line 2. 
Table 4

Results for 100 Satellites in a Spherical Volume around the Host

\begin{tabular}{|c|c|c|c|c|c|c|c|c|c|c|c|}
\hline $\begin{array}{l}\text { (1) } \\
\text { Galaxy }\end{array}$ & $\begin{array}{c}(2) \\
\text { Model }\end{array}$ & $\begin{array}{c}(3) \\
N_{\max }\end{array}$ & $\begin{array}{c}(4) \\
N_{\text {cor }}\end{array}$ & $\begin{array}{c}(5) \\
\mathrm{RD} \chi^{2}\end{array}$ & $\begin{array}{c}(6) \\
\Phi\end{array}$ & $\begin{array}{c}(7) \\
p_{\text {pos }}(\%)\end{array}$ & $\begin{array}{c}(8) \\
p_{\text {kin }}(\%)\end{array}$ & $\begin{array}{c}(9) \\
p_{\text {tot }}(\%)\end{array}$ & $\begin{array}{c}(10) \\
\sigma_{\|}(\mathrm{kpc})\end{array}$ & $\begin{array}{c}(11) \\
\sigma_{\perp}(\mathrm{kpc})\end{array}$ & $\begin{array}{c}(12) \\
\operatorname{L}_{\operatorname{Lax}} \\
\text { min }\end{array}$ \\
\hline & $\mathrm{z}_{\mathrm{r}} \mathrm{M}_{\max }$ & 32 & 21 & 1.01 & 63.4 & 0.3 & 11 & 0.033 & 203.7 & 13.4 & $0.69_{0.31}^{0.97}$ \\
\hline & $\mathrm{z}_{\mathrm{r}}$ & 32 & 21 & 1.25 & 63.4 & 0.07 & 11 & 0.0077 & 212.6 & 13.3 & $0.74_{0.31}^{1.01}$ \\
\hline \multirow[t]{5}{*}{ LGa } & $\mathrm{M}_{\max }$ & 32 & 20 & 0.61 & 52.2 & 7.1 & 21.5 & 1.5 & 138.5 & 12.8 & $0.72_{0.51}^{0.86}$ \\
\hline & $\mathrm{M}_{\mathrm{z}=0}$ & 30 & 20 & 0.58 & 69.3 & 20.65 & 9.8 & 2.0 & 152.6 & 13.1 & $0.57_{0.43}^{0.75}$ \\
\hline & $\mathrm{M}_{\mathrm{star}}$ & 27 & 16 & 0.63 & 52.1 & 93.41 & 44.2 & 41.3 & 174.2 & 11.8 & $0.75_{0.37}^{1.1}$ \\
\hline & $\mathrm{z}_{\mathrm{r}} \mathrm{M}_{\max }$ & 28 & 19 & 1.52 & 118.1 & 27.96 & 8.7 & 2.4 & 140.2 & 13.8 & $0.93_{0.6}^{1.26}$ \\
\hline & $\mathrm{Z}_{\mathrm{r}}$ & 28 & 19 & 1.53 & 118 & 21.55 & 8.7 & 1.9 & 140.2 & 13.7 & $0.93_{0.6}^{1.26}$ \\
\hline \multirow[t]{3}{*}{ LGb } & $\mathrm{M}_{\max }$ & 33 & 19 & 1.63 & 130.6 & 9.09 & 48.6 & 4.4 & 129.7 & 13.1 & $0.74_{0.39}^{0.99}$ \\
\hline & $\mathrm{M}_{\mathrm{z}=0}$ & 33 & 17 & 1.80 & 130.9 & 9.99 & 100 & 10.0 & 126.7 & 12.6 & $0.7_{0.46}^{0.9}$ \\
\hline & $\mathrm{M}_{\mathrm{star}}$ & 30 & 20 & 1.38 & 101.4 & 18.79 & 9.8 & 1.9 & 168 & 11.9 & $0.8_{0.63}^{1}$ \\
\hline
\end{tabular}

of rotation if seen edge-on and only the line-of-sight velocities are considered, with the opposite sides, respectively, receding and approaching. However, they do not qualify as "disks." We are well aware that our simulated planes of satellites are not perfect matches to M31's, but if they are of similar nature, then our results suggest that the observed VPoS of I13 is not a disk, and that one-third to one-half of its members should have large proper motions perpendicular to the plane (up to $200 \mathrm{~km} \mathrm{~s}^{-1}$ ). These high proper motion satellites are likely to be the most spatially and kinematically isolated ones, for instance, And I, And III, And IX, And XII, And XIV, and And XVI, because the more clustered satellites are likely to be one coherent group. We can only hope that the Hubble Space Telescope will live long enough to test this, or that future observatories will be able to perform such measurements. An alternative will be by combining future adaptive optic imaging with earlier Hubble Space Telescope imaging to get proper motions. Water maser observations with very long baseline interferometry have also been used to derive proper motions in the LG (Brunthaler et al. 2005; Darling 2011), but this method is likely inapplicable for most of M31's low-mass satellites because of their lack of gas or star formation.

\subsection{On Satellite Population Models}

The properties of the planes of satellites we find are strongly affected by the stellar mass model used. This simply reflects the fact that we do not understand star formation in the lowest mass satellites of M31 and MW. Any study into the significance of planar structures of satellites, such as those by Bahl \& Baumgardt (2013); Pawlowski et al. (2014); Ibata et al. (2014b), will face similar problems. Therefore future studies investigating disks of faint satellites (i.e., fainter than classical satellites, i.e., $M_{V}>-10$ ) should account for the modeling uncertainties by exploring various models (even crudely) as we do here. In the present paper, our modeling was carried out with simplicity in mind, and given the small size of our sample (two galaxies only, although resulting from carefully built constrained initial conditions) it is perilous to assess the validity of our models based on so little evidence. Nevertheless, we note that the most realistic and significant planes are obtained using the $\mathrm{Z}_{\mathrm{r}} \mathrm{M}_{\max }$ model. Should this be confirmed with additional LG simulations, this would suggest that an accurate modeling of the reionization of the LG as we have done here is an important ingredient to reproduce the properties of the LG's satellite systems, as was pointed out in Ocvirk \& Aubert (2011). On the other hand, this problem should not affect studies relying on the brightest satellites of MW-type galaxies. In particular, the over-abundance of diametrically opposed co-rotating satellites reported by Ibata et al. (2014a) involves large LMC-type objects, which will come out as the most luminous satellites in the majority of our models.

\section{CONCLUSIONS}

We have searched for planar structure in a high-resolution $N$-body smoothed particle hydrodynamics $\Lambda$ CDM simulation of the Local Group, containing a MW-M31 galaxy pair. We model the satellite populations using five different sets of very simple recipes, reproducing roughly the variety of models considered in the literature. We describe a method for finding the plane containing the maximum of satellites and validate it on the observed VPoS of M31 found by I13. The model satellite population's spatial distribution is strongly dependent on the model prescriptions, and so are the properties of the planar configurations we find.

Since the satellite systems of our simulated galaxies may be instrinsically different from the real M31 (for instance with respect to their radial distribution), we focus on quantifying the significance of the simulated planes, as their probability of occurring in a random population. This allows us to quantify the degree of structure, or non-randomness of the satellite systems, in a manner similar to I13. Applying this method to our satellite population models we attempt to compare the simulation to the observations, especially the plane of satellites of Andromeda (Ibata et al. 2013) found by PAndAS. We also consider two alternative volumes (one extended PAndAS and one spherical) in order to further explore the simulation. We find several cases (a total of seven) of planes which are very unlikely ( $<1 \%$ chance) to be random alignments, showing that the simulated satellite populations are indeed highly structured. Our best maximum plane has 14 of 27 satellites in a plane of $14.1 \mathrm{kpc}$ dispersion, among which 11 are co-rotating. However, the observed VPoS of M31 is slightly richer in satellites, has a stronger co-rotation, and is still slightly thinner, which makes it stand out as overall more exceptional than our simulated planes by a factor of 10 or more in significance. The most significant simulated planes tend to be obtained with the $\mathrm{z}_{\mathrm{r}} \mathrm{M}_{\max }$ model, highlighting the possibly important role of a realistic description of the inside-out reionization of the LG galaxies in investigations of the properties of its low-mass satellite systems. Most of the simulated planes consist of one coherent group 
containing about half of the plane's satellites and forming its backbone, aligning by chance with another group or several isolated, kinematically unrelated satellites. This is the main result of this study. Therefore, although the planes we find are generally dominated by one real structure, they are also partly fortuitous and are thus not kinematically coherent structures as a whole: one-third to one-half of their satellites will fly out of the plane on a short timescale $(\sim 150 \mathrm{Myr})$, although the main defining group may conserve its alignment and realign by chance with another set of satellites.

Provided that the simulated and observed planes of satellites are indeed of the same nature, our results suggest that the VPoS of M31 is not a coherent disk and that one-third to one-half of its satellites must have large proper motions perpendicular to the plane. We hope that future observational campaigns will be able to settle this debate.

This study was performed in the context of the EMMA (ANR-12-JS05-0001) and LIDAU project (ANR-09-BLAN0030), funded by the Agence Nationale de la Recherche (ANR). The RT computations were performed using HPC resources from GENCI-[CINES/IDRIS] (grant 2011-[x2011046667]), on the hybrid queue of titane at Centre de Calcul Recherche et Technologie, as well as Curie, during a grand challenge time allocation (project PICON: Photo-Ionisation of CONstrained realizations of the local group). The CLUES simulations were performed at the Leibniz Rechenzentrum Munich (LRZ) and at the Barcelona Supercomputing Center (BSC). A.K. is supported by the Ministerio de Economía y Competitividad (MINECO) in Spain through grant AYA2012-31101 as well as the ConsoliderIngenio 2010 Programme of the Spanish Ministerio de Ciencia e Innovación (MICINN) under grant MultiDark CSD2009-00064. He also acknowledges support from the Australian Research Council (ARC) grants DP130100117 and DP140100198. He further thanks Stella for l'idole des jaunes. The authors thank C. Scannapieco for precious hints dispensed in the initial phase of the project, as well as the CLUES collaborators for useful discussions. The authors thank D. Munro for freely distributing his Yorick programming language, ${ }^{6}$ and its yorick-gl extension which was used in the course of this study.

\section{REFERENCES}

Aubert, D., Pichon, C., \& Colombi, S. 2004, MNRAS, 352, 376

Aubert, D., \& Teyssier, R. 2008, MNRAS, 387, 295

Aubert, D., \& Teyssier, R. 2010, ApJ, 724, 244

Bahl, H., \& Baumgardt, H. 2014, MNRAS, 438, 2916

Bowden, A., Evans, N. W., \& Belokurov, V. 2013, MNRAS, 435, 928

Brook, C. B., Di Cintio, A., Knebe, A., et al. 2014, ApJL, 784, L14

Brown, T. M., Tumlinson, J., Geha, M., et al. 2014, ApJ, 796, 91

Brunthaler, A., Reid, M. J., Falcke, H., Greenhill, L. J., \& Henkel, C. 2005, Sci, 307, 1440

Busha, M. T., Alvarez, M. A., Wechsler, R. H., Abel, T., \& Strigari, L. E. 2010, ApJ, 710, 408
Collins, M. L. M., Chapman, S. C., Rich, R. M., et al. 2013, ApJ, 768, 172

Conn, A. R., Ibata, R. A., Lewis, G. F., et al. 2012, ApJ, 758, 11

Conn, A. R., Lewis, G. F., Ibata, R. A., et al. 2013, ApJ, 766, 120

Darling, J. 2011, ApJL, 732, L2

Deason, A. J., Belokurov, V., Evans, N. W., \& Johnston, K. V. 2013, ApJ, 763, 113

Deason, A. J., McCarthy, I. G., Font, A. S., et al. 2011, MNRAS, 415, 2607

Diaz, J. D., Koposov, S. E., Irwin, M., Belokurov, V., \& Evans, N. W. 2014, MNRAS, 443, 1688

Forero-Romero, J. E., Hoffman, Y., Yepes, G., et al. 2011, MNRAS, 417, 1434

Gill, S. P. D., Knebe, A., \& Gibson, B. K. 2004, MNRAS, 351, 399

Gnedin, N. Y. 2000, ApJ, 542, 535

Gottloeber, S., Hoffman, Y., \& Yepes, G. 2010, arXiv:1005.2687

Guo, Q., White, S., Angulo, R. E., et al. 2013, MNRAS, 428, 1351

Ibata, N. G., Ibata, R. A., Famaey, B., \& Lewis, G. F. 2014a, Natur, 511, 563

Ibata, R. A., Ibata, N. G., Lewis, G. F., et al. 2014b, ApJL, 784, L6

Ibata, R. A., Lewis, G. F., Conn, A. R., et al. 2013, Natur, 493, 62

Kang, X., Mao, S., Gao, L., \& Jing, Y. P. 2005, A\&A, 437, 383

Knebe, A., Libeskind, N. I., Doumler, T., et al. 2011a, MNRAS, 417, L56

Knebe, A., Libeskind, N. I., Knollmann, S. R., et al. 2011b, MNRAS, 412, 529

Knollmann, S. R., \& Knebe, A. 2009, ApJS, 182, 608

Koch, A., \& Grebel, E. K. 2006, AJ, 131, 1405

Koposov, S., Belokurov, V., Evans, N. W., et al. 2008, ApJ, 686, 279

Kroupa, P., Theis, C., \& Boily, C. M. 2005, A\&A, 431, 517

Kunkel, W. E., \& Demers, S. 1976, in Royal Greenwich Observatory Bulletins, Vol. 182, The Galaxy and the Local Group, ed. R. J. Dickens, J. E. Perry, F. G. Smith, \& I. R. King (Herstmonceaux: Royal Greenwich Observatory), 241

Libeskind, N. I., Cole, S., Frenk, C. S., Okamoto, T., \& Jenkins, A. 2007, MNRAS, 374, 16

Libeskind, N. I., Frenk, C. S., Cole, S., Jenkins, A., \& Helly, J. C. 2009, MNRAS, 399,550

Libeskind, N. I., Frenk, C. S., Cole, S., et al. 2005, MNRAS, 363, 146

Libeskind, N. I., Knebe, A., Hoffman, Y., Gottlöber, S., \& Yepes, G. 2011a, MNRAS, 418, 336

Libeskind, N. I., Knebe, A., Hoffman, Y., et al. 2011b, MNRAS, 411, 1525

Libeskind, N. I., Yepes, G., Knebe, A., et al. 2010, MNRAS, 401, 1889

Lunnan, R., Vogelsberger, M., Frebel, A., et al. 2012, ApJ, 746, 109

Lynden-Bell, D. 1976, MNRAS, 174, 695

Macciò, A. V., Kang, X., Fontanot, F., et al. 2010, MNRAS, 402, 1995

McConnachie, A. W., \& Irwin, M. J. 2006, MNRAS, 365, 902

Moster, B. P., Naab, T., \& White, S. D. M. 2013, MNRAS, 428, 3121

Ocvirk, P., \& Aubert, D. 2011, MNRAS, 417, L93

Ocvirk, P., Aubert, D., Chardin, J., et al. 2013, ApJ, 777, 51

Ocvirk, P., Gillet, N., Aubert, D., Chardin, J., et al. 2014, ApJ, 777, 51

Pawlik, A. H., Milosavljević, M., \& Bromm, V. 2013, ApJ, 767, 59

Pawlowski, M. S., Famaey, B., Jerjen, H., et al. 2014, MNRAS, 442, 2362

Pawlowski, M. S., \& McGaugh, S. S. 2014, ApJL, 789, L24

Sawala, T., Frenk, C. S., Fattahi, A., et al. 2014, arXiv:1404.3724

Shaya, E. J., \& Tully, R. B. 2013, MNRAS, 436, 2096

Spergel, D. N., Bean, R., Doré, O., et al. 2007, ApJS, 170, 377

Springel, V. 2005, MNRAS, 364, 1105

Springel, V., \& Hernquist, L. 2003, MNRAS, 339, 289

van der Marel, R. P., Fardal, M., Besla, G., et al. 2012, ApJ, 753, 8

Watkins, L. L., Evans, N. W., \& An, J. H. 2010, MNRAS, 406, 264

Yepes, G., Gottlöber, S., \& Hoffman, Y. 2014, NewAR, 58, 1

Yniguez, B., Garrison-Kimmel, S., Boylan-Kolchin, M., \& Bullock, J. S. 2014, MNRAS, 439, 73

Zentner, A. R., Kravtsov, A. V., Gnedin, O. Y., \& Klypin, A. A. 2005, ApJ, 629,219

6 http://www.maumae.net/yorick/doc/index.html 